\title{
A previously unreported potential malaria vector in a dry ecology of Kenya
}

\author{
Edwin O. Ogola, Edith Chepkorir, Rosemary Sang and David P. Tchouassi ' (D)
}

\begin{abstract}
Background: In Kenya, malaria remains a major public health menace equally affecting the semi-arid to arid ecologies. However, entomologic knowledge of malaria vectors in such areas remains poor.

Methods: Morphologically-identified wild-caught Anopheles funestus (s.l.) specimens trapped outdoors from the semi-arid to arid area of Kacheliba, West Pokot County, Kenya, were analysed by PCR and sequencing for species identification, malaria parasite infection and host blood-meal sources.

Results: Three hundred and thirty specimens were analysed to identify sibling species of the An. funestus group, none of which amplified using the available primers; two were infected with Plasmodium falciparum and Plasmodium ovale, separately, while $84 \%(n=25)$ of the blood-fed specimens had fed on humans. Mitochondrial cytochrome c oxidase subunit 1 (cox1) and nuclear ribosomal internal transcribed spacer 2 (ITS2) sequences of 55 specimens (Plasmodium-positive, blood-fed and Plasmodium-negative) did not match reference sequences, possibly suggesting a previously unreported species, resolving as two clades.
\end{abstract}

Conclusions: Our findings indicate the existence of yet-to-be identified and described anopheline species with a potential as malaria vectors in Kenya.

Keywords: Anopheles funestus group, Malaria transmission, Entomological surveillance, Molecular approaches, Dry ecology, Kenya

\section{Background}

Entomological surveillance remains integral to effective intervention strategies towards malaria elimination. Previous studies have highlighted the importance of applying molecular approaches including sequencing in malaria entomologic surveillance, particularly involving species among known vector complexes as in the Anopheles funestus group [1-3]. This way, the identity and distribution of the species including their bionomic roles in malaria transmission can be accurately and comprehensively determined to guide appropriate measures in their control [3].

Anopheles funestus mosquitoes have been previously reported in semi-arid to arid areas of Kenya [4, 5]; however, these studies were not supported by molecular data. While An. funestus (s.s.) is the most ubiquitous species in the An. funestus group, our recent findings

\footnotetext{
* Correspondence: dtchouassi@icipe.org

International Centre of Insect Physiology and Ecology (icipe), P.O. Box 30772-00100, Nairobi, Kenya
}

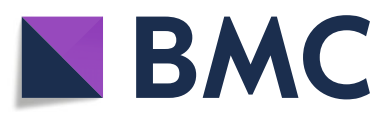

(c) The Author(s). 2019 Open Access This article is distributed under the terms of the Creative Commons Attribution 4.0 International License (http://creativecommons.org/licenses/by/4.0/), which permits unrestricted use, distribution, and

reproduction in any medium, provided you give appropriate credit to the original author(s) and the source, provide a link to the Creative Commons license, and indicate if changes were made. The Creative Commons Public Domain Dedication waiver (http://creativecommons.org/publicdomain/zero/1.0/) applies to the data made available in this article, unless otherwise stated. 
monitoring project in Kacheliba $\left(1^{\circ} 29^{\prime} 81^{\prime \prime} \mathrm{N}, 35^{\circ} 01^{\prime} 80^{\prime \prime} \mathrm{E}\right)$, West Pokot County (close to Uganda), were used in this study. The samples were collected outdoors in May 2016 using $\mathrm{CO}_{2}$-baited BG Sentinel traps (note: indoor collections using aspiration yielded none of this species). The weather in this area is hot and dry most of the year, with annual temperatures averaging about $21{ }^{\circ} \mathrm{C}$. Rainfall is usually scarce and irregular from one year to the next with annual mean values ranging from about 300 to 400 $\mathrm{mm}$. The mean daily rainfall during the sampling period in May 2016 was $5.55 \mathrm{~mm}$. The main human activity in the area is nomadic pastoralism [5]. The common livestock in the area include cattle, goats and sheep.

\section{DNA extraction and identification of sibling species in the} Anopheles funestus group

Genomic DNA extracted from individual whole specimens using Qiagen DNeasy Blood and Tissue Kit (Qiagen, Hilden, Germany) was used to identify by PCR the sibling species as well as probing for Plasmodium infection and host blood-meal sources. We identified species of the An. funestus group using an established cocktail of primers [10, 11], as previously described [3]. Briefly, PCRs were conducted on a SimpliAmp Thermal Cycler (Applied Biosystems, Loughborough, UK) in a $15 \mu \mathrm{l}$ reaction volume containing $0.5 \mu \mathrm{M}$ each of the primers, 3 $\mu \mathrm{l}$ of $5 \times$ Hot Firepol Blend Master Mix Ready to Load (Solis BioDyne, Tartu, Estonia) and $2 \mu$ of DNA template. The cycling parameters were: initial denaturation at $95{ }^{\circ} \mathrm{C}$ for $15 \mathrm{~min}$, followed by 30 cycles of denaturation at $95{ }^{\circ} \mathrm{C}$ for $30 \mathrm{~s}$, annealing at $46{ }^{\circ} \mathrm{C}$ for $30 \mathrm{~s}$ and extension at $72{ }^{\circ} \mathrm{C}$ for $40 \mathrm{~s}$, and a final extension at $72^{\circ}$ $\mathrm{C}$ for $10 \mathrm{~min}$. Size fragments characteristic of each species were scored after separation in agarose gel electrophoresis $(1.5 \%)$ stained with ethidium bromide against a 100 bp DNA ladder (O' Gene Ruler, Fermentas, Fisher Scientific, Loughborough, UK).

\section{Detection of Plasmodium malaria parasites}

Individual samples were tested for Plasmodium infection by analyzing high resolution melting (HRM) profiles generated from real time-PCR (RT-PCR) products of non-coding mitochondrial sequence (ncMS) [12] and/or amplification of the cytochrome $c$ oxidase subunit 1 (cox1) gene [13] as previously described [3]. Plasmodium falciparum DNA obtained from National Institute for Biological Standards and Control (NIBSC; London, UK) was used as a positive control. Conventional PCR for Plasmodium spp. detection targeting the cox1 gene was carried out in a $15 \mu \mathrm{l}$ reaction containing $0.5 \mu \mathrm{M}$ of each primer, $9 \mu \mathrm{l}$ of PCR water, $3 \mu \mathrm{l}$ of $5 \times \mathrm{Hot} \mathrm{Firepol}^{\circ}$ Blend Master Mix (Solis BioDyne) and $2 \mu \mathrm{l}$ of DNA template. The amplifying conditions were: $95{ }^{\circ} \mathrm{C}$ for $15 \mathrm{~min}$, followed by 40 cycles of $95{ }^{\circ} \mathrm{C}$ for $30 \mathrm{~s}, 59{ }^{\circ} \mathrm{C}$ for $30 \mathrm{~s}$ and $72{ }^{\circ} \mathrm{C}$ for $40 \mathrm{~s}$, and a final extension at $72{ }^{\circ} \mathrm{C}$ for 10 min. PCR product of all amplicons were purified using ExoSAP-IT (USB Corporation, Cleveland, OH, USA) and outsourced for sequencing (Macrogen, Seoul, South Korea).

We further confirmed the species identity of Plasmodium sporozoite positive mosquito specimens by amplifying and sequencing of ribosomal DNA internal transcribed spacer region 2 (rDNA ITS2) [14] and/or the mitochondrial cox1 gene [15] as described previously [3]. We also amplified and sequenced 28 randomly selected specimens found negative for Plasmodium infection. The amplicons were purified as reported previously and outsourced for bidirectional sequencing (Inqaba Biotech, Pretoria, South Africa).

\section{Blood-meal analyses}

PCR targeting the genes cytochrome $b$ (cytb), $16 S$ ribosomal rRNA and cox1 were used to detect blood-meal host sources from the engorged specimen Anopheles by RT-PCR-HRM (Rotor Gene Q thermo cycler; Qiagen) and compared to profiles of known controls (positive: cow, Swiss mouse, pig, goat, chicken and human; negative: DNA from sugar-fed insectary-reared Anopheles gambiae) as described previously [3]. High resolution melting profiles generated were analyzed using HRM analysis tools present in the RGQ software (Qiagen). Vertebrate hosts were determined through comparison of the blood-meal HRM melt profiles to those of the standard reference control species.

\section{Sequence and statistical analyses}

Mosquito and Plasmodium sequences were viewed and edited in Chromas, embedded in MEGA v.6 software [16] prior to querying the GenBank using BLASTn. Multiple sequence alignments of the resulting contiguous sequences (mosquito or Plasmodium) were performed using ClustalW in MEGA v.6 with default parameters. For mosquito sequences, maximum likelihood (ML) trees were constructed with nodal support for the different groupings evaluated through 1000 bootstrap replications utilizing the GTR+G and Jukes and Cantor model of sequence evolution for $\operatorname{cox} 1$ and ITS fragments, respectively. For ITS, indels were excluded from analysis. We further estimated the percent evolutionary divergence between the species found and reference species, including those of the An. funestus group, in MEGA v.6. The human blood index is expressed as the proportion of blood-feeding on humans of the total number of blood-fed mosquitoes examined.

\section{Results and discussion}

We analyzed a total of 330 morphologically-identified An. funestus (s.l.) specimens, none of which amplified 
using the established ITS2 cocktail primers. Plasmodium infection was detected in two specimens. Analysis of the resultant $162 \mathrm{bp}$ each of the sequenced isolates of the ncMS gene followed by Blastn searches in GenBank showed one of them as having $100 \%$ identity to P. falciparum (GenBank: CP017005) and the other with 100\% identity to P. ovale (GenBank: AB354571).

Ribosomal ITS2 and mitochondrial $\operatorname{cox} 1$ sequences of the two PCR-Plasmodium-positive mosquito specimens could not be matched to reference anopheline sequences or known vector species in GenBank, BOLD or VectorBase databases (Fig. 1), suggesting the existence of a potentially important unreported malaria vector species.

Sequences of 25 blood-fed mosquito specimens (both ITS2 and cox 1 ) were identical to those found Plasmodium-positive. Interestingly, a higher proportion of the blood-fed samples $(21 / 25)$ had taken a blood meal from humans with an estimated human blood index of $84 \%$ for this previously unreported species. The remainder had fed on goats. Inclusion of sequences from selected
Plasmodium-negative specimens in a phylogenetic tree, show that the species resolves as two well supported clades (Fig. 1) although with clade differences in the representation of the samples between the ITS and cox 1 genes. The Plasmodium-positive specimens fell into a single clade for ITS and into each of the two clades for cox1 (Fig. 1). Select sequences of the mosquito specimens are available on GenBank: cox1 (528-646 nt; GenBank: MK047664-MK047672) and ITS2 (540-538 nt; GenBank: MK043038-MK043040). The species share 96\% identity to the Anopheles sp. AGB-2016 (GenBank: KU948655), reported in Al'Sadah, Yemen. The next most closely related sequences (92\% identity) are from other anopheline mosquito species (Fig. 1).

The bionomic traits uncovered for the species found in the present study, being trapped outdoors and with a high proportion feeding on humans, may compromise effective malaria control using the currently used indoor vector control tools, namely insecticide-treated bednets or indoor residual spraying. This species may contribute

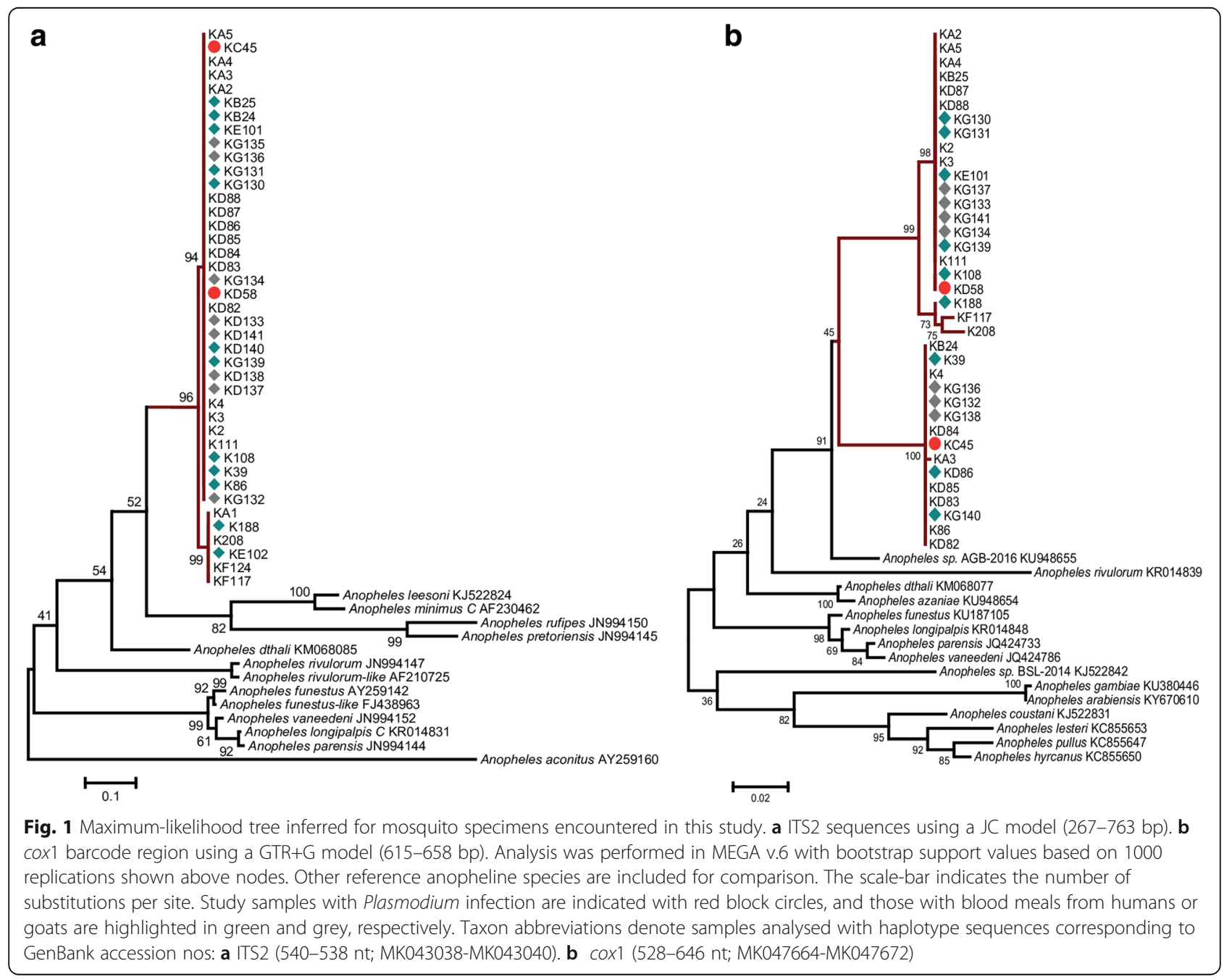




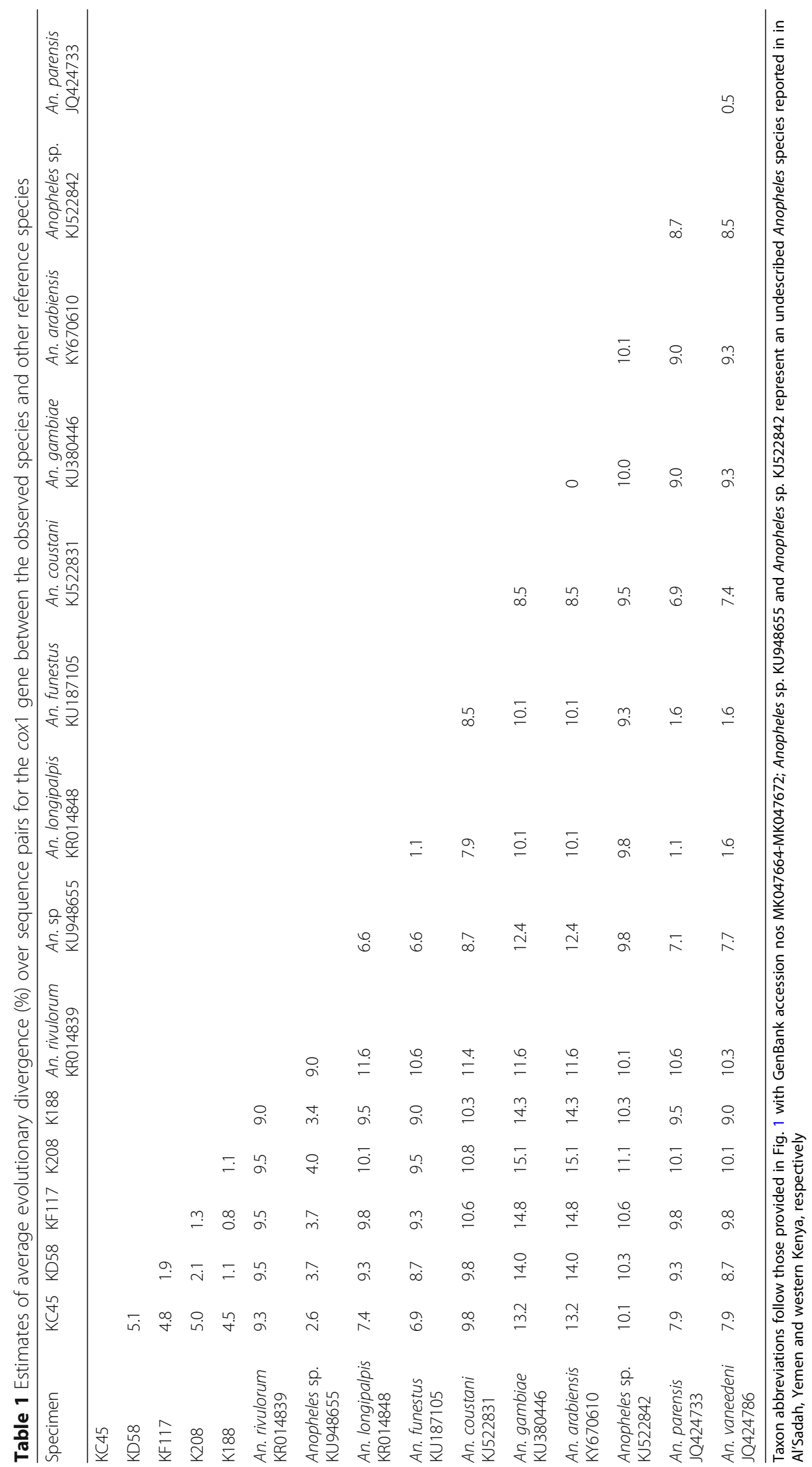


to stealth transmission and possibly sustenance of malaria in this focus. Our findings highlight the importance of employing molecular methods in entomological surveillance which target more than just the known malaria vectors. Furthermore, daytime activity is suggested for this species given that it was trapped using BG sentinel traps baited with $\mathrm{CO}_{2}$ set from 6:00 h to 18:00 h. A similar exhibition of such diurnal habit has recently been observed for An. funestus in West Africa; however, this was attributed to the scaling-up of universal coverage with long-lasting insecticidal nets [17]. Such a behaviour pattern could contribute to an increased risk of residual outdoor transmission, clearly representing a challenge for malaria control.

The specimens were morphologically identified as $A n$. funestus (s.l.), although analysis via a molecular approach showed much divergence from sibling species in the group which are known to occur in Kenya. This finding, in part, highlights a taxonomic challenge in accurately identifying species morphologically [18]. Support against molecular evidence for mosquito species delineation has been highlighted [19] on account of sequence variation among individuals of the same species. However, molecular analysis has been highly instrumental in uncovering divergence among morphologically indistinguishable cryptic species $[3,20]$. In fact, the DNA barcode targeting the cox 1 gene has been widely used for mosquito species identification including discrimination for cryptic species groups, in conjunction with the ITS2 region [21]. Based on the barcode region, previous studies have suggested an evolutionary divergence of 2$3 \%$ as a threshold for intraspecific variation [22-25]. The average evolutionary divergence over sequence pairs between the putative species and any other species ranged from 0.5 to $15.1 \%$ for the cox 1 sequence (Table 1 ). Observed levels of evolutionary divergence between this species and those in the Funestus group including other well-known malaria vectors in Kenya (An. gambiae, An. arabiensis, An. coustani [26]), surpasses this threshold, suggesting a separate genetic entity. Nonetheless, both morphological and molecular identification are useful for a detailed taxonomic elucidation of a given species important for vector surveillance and biodiversity studies $[1,27]$. As such, further morphological descriptions and ecological studies of this species are warranted. It may also be important to link the molecular forms to iso-female lines that can be used to provide information on genetic variation within families. Our detection of Plasmodium infection in the newly-detected Anopheles species potentially implicates it in malaria transmission. However, whether the parasite would have been transmitted or not cannot be conclusively answered based on our analysis but its potential role as secondary vector in the more arid areas of Kenya should be further investigated. Confirming its role as a vector may benefit from additional studies to detect human Plasmodium sporozoites through dissection of the salivary glands.

Anopheles funestus has been known to have the widest distribution range among the Funestus group [28]. Its lack of detection including other previously reported species in the group from this drought-prone ecology is intriguing. Rainfall in this semi-arid to arid area of northern Kenya is often scarce and irregular from one year to the next [29]. Previous findings have suggested that An. funestus can inhabit extreme dry conditions in the Sahel depending on the availability of suitable breeding sites such as man-made irrigation zones [30,31]. On the other hand, the influence of prolonged severe drought on population decline and elimination of $A n$. funestus from parts of Africa has been reported [32], suggesting that extreme climate variability can affect the survival of this species. The close relatedness of the species encountered in this study to that from the Arabian Peninsula raises the possibility of adaptation of this and related species to very dry ecologies. While it may be inconclusive as to whether An. funestus and other known sibling species occur in Kacheliba given the limited duration of sampling, further studies over a longer period are encouraged to investigate the species composition and adaptation of mosquitoes in the Funestus group in this ecology.

\section{Conclusions}

We have uncovered a species with potential as a malaria vector supported by sequence data and the exhibition of important bionomic traits such as the ability to feed on humans and that it was found infected with Plasmodium malaria parasites. The findings indicate the existence of yet-to-be identified and described anopheline species with potential as a malaria vector in the dry ecology of Kenya. More detailed studies including taxonomic description of the species and its ecological dynamics should be a focus of additional research.

\begin{abstract}
Abbreviations
CDC: Centers for Disease Control and Prevention; cox1: Cytochrome $C$ oxidase subunit 1; HRM: High resolution melting; PCR: Polymerase chain reaction; s.l.: Sensu lato; s.s.: Sensu stricto
\end{abstract}

\section{Acknowledgements}

We thank the community members in Kacheliba for providing access to their homesteads.

\section{Funding}

The study was funded by a grant from the Foundation for the National Institutes of Health through the Vector-Based Transmission of Control: Discovery Research (VCTR) programme of the Grand Challenges in Global Health initiative. Additionally, we gratefully acknowledge the financial support for this research by the following organizations and agencies: UK Department for International Development (DFID), Swedish International Development Cooperation Agency (Sida), Swiss Agency for Development and Cooperation (SDC), and the Kenyan Government. The views expressed herein do not necessarily reflect the official opinion of the donors. 


\section{Availability of data and materials}

Data supporting the conclusions of this article are included within the article. The datasets used and/or analyzed during the present study can be made available by the corresponding author upon reasonable request. Select sequences of the mosquito specimens were submitted to the GenBank database: cox1 (528-646 nt; MK047664-MK047672); ITS2 (540-538 nt; MK043038-MK043040).

\section{Authors' contributions}

DPT conceived the experiments. EC and RS performed sample collection and morphological identification. EOO and DPT conducted the experiments and analysed the results. DPT wrote the draft manuscript. All authors read and approved the final manuscript.

\section{Ethics approval and consent to participate}

Approval for mosquito trapping in households was sought from the Scientific and Ethical Review Unit (SERU), of the Kenya Medical Research Institute (protocol number KEMRI-SERU 2787). We also sought informed consent from village elders and household heads.

\section{Consent for publication}

Not applicable.

\section{Competing interests}

The authors declare that they have no competing interests.

\section{Publisher's Note}

Springer Nature remains neutral with regard to jurisdictional claims in published maps and institutional affiliations.

Received: 18 October 2018 Accepted: 31 January 2019

Published online: 11 February 2019

\section{References}

1. Lobo NF, Laurent BS, Sikaala CH, Hamainza B, Chanda J, Chinula D, et al. Unexpected diversity of Anopheles species in eastern Zambia: implications for evaluating vector behavior and interventions using molecular tools. Sci Rep. 2015;5:17952.

2. Laurent BS, Cooke M, Krishnankutty SM, Asih P, Mueller JD, Kahindi S, et al. Molecular characterization reveals diverse and unknown malaria vectors in the western Kenyan highlands. Am J Trop Med Hyg. 2016;94:327-35.

3. Ogola EO, Fillinger U, Ondiba IM, Villinger J, Masiga DK, Torto B, et al. Insights into malaria transmission among Anopheles funestus mosquitoes, Kenya. Parasit Vectors. 2018;11:577.

4. Mala AO, Irungu LW, Shililu Jl, Muturi EJ, Mbogo CM, Njagi JK, et al. Plasmodium falciparum transmission and aridity: a Kenyan experience from the dry lands of Baringo and its implications for Anopheles arabiensis control. Malar J. 2011;10:121,

5. Chepkorir E, Venter M, Lutomiah J, Mulwa F, Arum S, Tchouassi D, et al. The occurrence, diversity and blood feeding patterns of potential vectors of dengue and yellow fever in Kacheliba, West Pokot County, Kenya. Acta Trop. 2018;186:50-7.

6. Kaindoa EW, Matowo NS, Ngowo HS, Mkandawile G, Mmbando A, Finda M, et al. Interventions that effectively target Anopheles funestus mosquitoes could significantly improve control of persistent malaria transmission in south-eastern Tanzania. PLoS One. 2017;12:e0177807.

7. Kiuru CW, Oyieke FA, Mukabana WR, Mwangangi J, Kamau L, Muhia-Matoke D. Status of insecticide resistance in malaria vectors in Kwale County, coastal Kenya. Malar J. 2018;17:3.

8. Mutero C, Mutinga M, Ngindu A, Kenya P, Amimo F. Visceral leishmaniasis and malaria prevalence in West Pokot District, Kenya. East Afr Med J. 1992; 69:3-8.

9. Gillies M, Coetzee M. A supplement to the Anophelinae of Africa south of the Sahara (Afrotropical region). Johannesburg: The South African Institute for Medical Research; 1987.

10. Koekemoer L, Kamau L, Hunt R, Coetzee M. A cocktail polymerase chain reaction assay to identify members of the Anopheles funestus (Diptera: Culicidae) group. Am J Trop Med Hyg. 2002;66:804-11.

11. Cohuet A, Simard F, Toto J, Kengne P, Coetzee M, Fontenille D. Species identification within the Anopheles funestus group of malaria vectors in
Cameroon and evidence for a new species. Am J Trop Med Hyg. 2003;69: 200-5.

12. Chaumeau V, Andolina C, Fustec B, Ndam NT, Brengues C, Herder S, et al. Comparison of the performances of five primer sets for the detection and quantification of Plasmodium in anopheline vectors by real-time PCR. PLoS One. 2016;11:e0159160.

13. Echeverry DF, Deason NA, Makuru V, Davidson J, Xiao H, Niedbalski J, et al. Fast and robust single PCR for Plasmodium sporozoite detection in mosquitoes using the cytochrome oxidase I gene. Malar J. 2017;16:230.

14. Beebe NW, Saul A. Discrimination of all members of the Anopheles punctulatus complex by polymerase chain reaction-restriction fragment length polymorphism analysis. Am J Trop Med Hyg. 1995;53:478-81.

15. Folmer $\mathrm{O}$, Black $\mathrm{M}$, Hoeh W, Lutz R, Vrijenhoek R. DNA primers for amplification of mitochondrial cytochrome c oxidase subunit I from diverse metazoan invertebrates. Mol Marine Biol Biotechnol. 1994;3:294-9.

16. Tamura K, Stecher G, Peterson D, Filipski A, Kumar S. MEGA6: Molecular Evolutionary Genetics Analysis version 6.0. Mol Biol Evol. 2013;30:2725-9.

17. Sougoufara S, Diédhiou SM, Doucouré S, Diagne N, Sembène PM, Harry M, et al. Biting by Anopheles funestus in broad daylight after use of long-lasting insecticidal nets: a new challenge to malaria elimination. Malar J. 2014;13: 125.

18. Harbach R. The classification of genus Anopheles (Diptera: Culicidae): a working hypothesis of phylogenetic relationships. Bull Entomol Res. 2004;94 537-53.

19. Erlank E, Koekemoer LL, Coetzee M. The importance of morphological identification of African anopheline mosquitoes (Diptera: Culicidae) for malaria control programmes. Malar J. 2018;17:43.

20. Burke A, Dandalo L, Munhenga G, Dahan-Moss Y, Mbokazi F, Ngxongo S, et al. A new malaria vector mosquito in South Africa. Sci Rep. 2017;7:43779.

21. Beebe NW. DNA barcoding mosquitoes: advice for potential prospectors. Parasitology. 2018;145:622-33.

22. Hebert PD, Cywinska A, Ball SL. Biological identifications through DNA barcodes. Proc R Soc Lond B Biol Sci. 2003;270:313-21.

23. Meyer CP, Paulay G. DNA barcoding: error rates based on comprehensive sampling. PLoS Biol. 2005;3:e422.

24. Cywinska A, Hunter F, Hebert PD. Identifying Canadian mosquito species through DNA barcodes. Med Vet Entomol. 2006;20:413-24.

25. Tchouassi DP, Bastos AD, Sole CL, Diallo M, Lutomiah J, Mutisya J, et al. Population genetics of two key mosquito vectors of Rift Valley fever virus reveals new insights into the changing disease outbreak patterns in Kenya. PLoS Negl Trop Dis. 2014;8:e3364.

26. Mwangangi JM, Muturi EJ, Muriu SM, Nzovu J, Midega JT, Mbogo C. The role of Anopheles arabiensis and Anopheles coustani in indoor and outdoor malaria transmission in Taveta District, Kenya. Parasit Vectors. 2013;6:114.

27. Teletchea F. After 7 years and 1000 citations: comparative assessment of the DNA barcoding and the DNA taxonomy proposals for taxonomists and non-taxonomists. Mitochondrial DNA. 2010;21:206-26.

28. Dia I, Guelbeogo MW, Ayala D. Advances and perspectives in the study of the malaria mosquito Anopheles funestus. In: Manguin S, editor. Anopheles Mosquitoes. New Insights into Malaria Vectors. London: Intech; 2013. p. 828.

29. MoALF. Climate risk profile for West Pokot. Kenya County Climate Risk Profile Series. Nairobi: The Kenya Ministry of Agriculture, Livestock and Fisheries (MoALF); 2016. p. 21.

30. Konate L, Diop A, Sy N, Faye M, Deng Y, Izri A, et al. Comeback of Anopheles funestus in Sahelian Senegal. Lancet. 2001;358:336.

31. Labbo R, Fouta A, Jeanne I, Ousmane I, Duchemin J. Anopheles funestus in Sahel: new evidence from Niger. Lancet. 2004;363:660.

32. Mouchet J, Faye O, Julvez J, Manguin S. Drought and malaria retreat in the Sahel West Africa. Lancet. 1996;348:1735-6. 